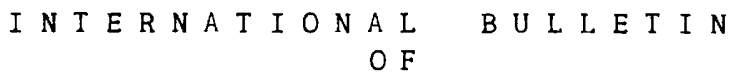

B A C TERIOLOGICAL NOMENCLA TURE

A N D

I A X O N O MY

Volume 7

January 15, 1957

No. 1

\title{
BIOCHEMICAL TESTS FOR THE \\ DIFFERENTIATION OF KLEBSIELLA AND CLOACA
}

E. Hormaeche ${ }^{1}$ and M. Munilla

The latest report (1) of the Enterobacteriaceae Subconmittee (1953) defines the Klebsiella group, (including Aerobacter aerogenes) as made up of nonmotile, indole-negative, MR-negative, VP-positive, citrate-positive, non-gelatin-liquefying, Gram-negative rods. They also decompose urea, although their urease activity is much weaker than that of Proteus. While all these biochemical characters are considered typical of the group, the report also mentions that included in the group are individual cultures which give aberrant results in one or more of these tests. Although it was realized that motile and nonmotile strains had many characters in common, as the Subcommittee eliminated motile strains from Klebsiella, it was possible to correlate motility with biochemical behaviour. There are, therefore, sound reasons for placing these motile strains in a special group of their own, the Cloaca group.

Brooke (2) compared 108 cultures of cloaca, 71 of them motile, with 303 nonmotile Klebsiella strains. He found that all strains of Cloaca liquefied gelatin, none produced gas from glycerol, one fermented inositol, one dulcitol, 20 were urea-positive and only 9 promptly fermented salicin. All 303 Klebsiella cultures produced gas from glycerol, 296 promptly fermented inositol, 91 dulcitol, 17 liquefied gelatin, 300 were urea-positive and 302 fermented salicin in 24 hours. He concluded that gelatin liquefaction, motility, inability to produce gas from glycerol, and to ferment inositol, are the best characters to identify Cloaca, while dulcitol, salicin and urea tests may also be useful. His cultures of Klebsiella included 13 MR-positive strains, 12 indole producers, and 1 VP-negative strain. Cellobiose and starch were not used by Brooke. For the urea test he used the Christensen medium as modified by Kristensen, with phenol red indicator as recommended by Kauffmann (3). Moeller (4) in his excellent paper

\footnotetext{
${ }^{1}$ Medical Center Laboratorles, State Insurance Bank, Montevideo, Uruguay.
}

(Page 1) 
on the biochemical behaviour of the Enterobacteriaceae included 89 strains of Klebsiella, one of them motile, and 27 cultures of Cloaca, 2 of them "O" forms. Both groups fermented cellobiose and most of them were positive in the KCN test. As distinguishing characters he found that 100 per cent of the klebsiellae fermented inositol, but in contrast with Brooke's results, so did 26 per cent of the strains of Cloaca; neither glycerol nor starch was used. Adonitol was fermented by 94 per cent of Moeller's Klebsiella and 26 per cent of Cloaca strains. Gelatin was liquefied by 11 per cent of Klebsiella strains, but 11 per cent of Cloaca were negative. With salicin his figures are 99 per cent positive for Klebsiella and 100 per cent for Cloaca in three days, and with dulcitol 26 per cent and 11 per cent respectively. For the urea test he used the same medium as Brooke and found 87 per cent positive in Klebsiella and 63 per cent in Cloaca. Therefore it appears that there is no strict correlation between motility and any of these tests, gelatin liquefaction remaining as the best single test for differentiation of the two groups.

In a later paper Moeller (5) mentions the results of his improved and simplified tests for decarboxylases, 86 Klebsiella and $27 \mathrm{Cloaca}$ strains were tested. The first were all negative in the arginine test and 2 per cent positive with ornithine; cultures of Cloaca were respectively 93 per cent and 96 per cent positive. All gave negative tests with glutamic acid, and with lysine all strains of Klebsiella were positive while those of Cloaca were 52 per cent positive, including delayed and weak reactions. Apparently thus far, arginine and ornithine tests are best for distinguishing the two groups.

Edwards and Fife (6) tested 128 motile and 626 nonmotile cultures. Of the motile, 61 per cent fermented inositol, 51 per cent adonitol, 58 per cent produced gas from glycerol (including delayed reactions), 28 per cent were urea-positive, 21 per cent fermented dulcitol and 99.3 per cent salicin; the positive tests from the second group were respectively 98 , $92,92,92,30$ and 99.6 per cent. With soluble starch 1.9 per cent of motile cultures produced gas in four days, while 61 per cent fermented it without gas, against 99 per cent nonmotile fermenting 1 t and 82 per cent producing gas. Gelatin was liquefied by 99 motile and by 28 nonmotile strains. Cellobiose and KCN tests were not used. The authors arrive at the conclusion that no single biochemical test or combination of tests is clearly sufficlent to distinguish Klebslella from Cloaca, and that many aberrant and intermediate strains occur. 
They consider motility and gelatin liquefaction to be the best distinguishing characters, but they think that among their nonmotile strains there may be some "O" forms of Cloaca, which seems probable on the basis of their results.

At present the situation is confusing since there exist such discrepancies concerning the value of all known criteria, and Moeller's decarboxylase tests have not yet, to our knowledge, been confirmed by others. The classification of many strains may present insurmountable difficulties and there is even a certain reluctance to admit as Klebsiella some of the serotypes considered as aberrant, either because of their IMViC reactions or because they liquefy gelatin. While admittedly the problem is far from solved, it is our purpose to show in this paper which tests we belleve to be the best. By their use it is possible and even easy for us to classify by their biochemical behaviour most strains with which we have to deal, although there remain some "intermediates" as is to be expected. We would like to add that in our opinion distinguishing between Klebsiella and Cloaca is not as difficult as between them and the related groups, Escherichia coli, E. freundii, and Serratia, a subject which we intend to discuss in a later paper.

\section{MATERIAL}

The 72 Klebsiella cultures representing the accepted serological capsular types mentioned in Table 1 were obtalned from Drs. F. Kauffmann and P. R. Edwards; to whom we express our gratitude. All strains in Table 2 were isolated by us. Dr. Edwards sent us at our request many motile and nonmotile strains which are not included in our tables. With the exception of 43 motile cultures from Dr. Edwards, all the remaining strains in Table 3 were isolated in Montevideo, most of them from human stools, many from other human materials and many also from cow's milk and from water. Not included in our tables are 2 nonmotile and 6 motile anaerogenic strains, which we have left out as they would oblige us to tabulate them separately. They are of no importance in this study as they behaved like the aerogenic types. Fifteen more anaerogenic, motile cultures resembling Cloaca were classified as Serratia on the basis of their behaviour with respect to gelatin liquefaction, and milk peptonization, and to cellobiose, rhamnose and arginine tests. We would also like to thank 
Dr. Cowan, curator of the National Collection of Type Cultures, for sending us many cultures used as reference and $\mathrm{Dr}$. V. Moeller for testing about 40 of our cultures for decarboxylases.

\section{METHODS}

Motility was determined both by direct observation in hanging drops of young broth cultures, and by the use of semisolid medium as recommended by Edwards and Brunner. Nonmotile forms showing the biochemical characters of Cloaca were kept in the semisolid medium for months both at $37^{\circ} \mathrm{C}$. and at room temperature with periodic transfers. In this way we obtained motile cultures on several occasions. For capsule observation we employed the Burri method as recommended by the author for Splrochaeta with Gunther Wagner "Pellikan 541" India ink.

The fermentation of carbohydrates was tested in broth prepared with beef extract, using the local product from the Frigorifico Nacional 0.5 per cent, Evans' peptone 1.0 per cent, and $\mathrm{NaCl} 0.5$ per cent. The $\mathrm{pH}$ was adjusted to 7.2-7.4 and phenol red used as indicator. With the exception of lactose, glucose, starch and glycerol, which were used at 1 per cent, all carbohydrates were added in the proportion of 0.5 per cent, Seltz filtered, and all tubes were fitted with gas bells, as it is our experience that to observe gas production from glucose only in misleading. Some strains anaerogenic in glucose produce gas from a different carbohydrate; while transfers made from tubes showing gas are frequently gas positive in glucose, this is not always the case. Tubes were cork stoppered and kept under observation for one month, although we do not think it necessary to observe them longer than 15 days, save in the case of milk and gelatin. Gas production from glycerol was read after four days.

For starch we followed the recommendation of Levine (7) who, as far back as 1918 used nonsoluble starch and rightly advocated its usefulness for the differentiation of Klebsiella and Cloaca. To avoid Iumps and assure uniformity of the medium the starch is finely ground, added to cold water, the mixture stirred and boiled 5 minutes. The resulting jelly is added to the basic medium, distributed in sterilized tubes fitted with gas bells, and sterlilized at $110^{\circ} \mathrm{C}$. for 15 minutes. The tubes were inoculated with a loopful of 24-hour broth 
culture, observed daily, and if on the fourth day there was no trace of gas in the bell, a red-hot wire loop was inserted into the medium. In this way we had some positive reactions when no gas was visible, and in doubtful cases we found it convenient to make transfers to new tubes where gas production was generally much quicker. Soluble starch is not so satisfactory as it is mostly dextrin. The results with dextrin are tabulated with the other tests.

The aesculin test was also performed according to Levine, with slight modifications. Bacto-peptone 0.5 per cent, and $\mathrm{K}_{2} \mathrm{HPO}_{4} \quad 0.1$ per cent, was used as the base. After sterilization, aesculin 0.3 per cent and iron citrate 0.05 per cent, Seitz filtered, were added, and the medium distributed in small tubes without gas bells. Positive reactions were recognized by an intense black colour, while no change or different shades of brown were considered as negative. Readings must take place after no more than 24 hours of incubation, to obtain the best differentiation.

For the MR and VP reactions the medium as recommended by Kauffmann (3) was employed, with the exception that we used Witte instead of Orthana peptone. For the VP test the Barritt method was used, readings being taken after 2 and 4 days. MR was tested after 4 days at $37^{\circ} \mathrm{C}$. For citrate utilization we used the Koser formula. Litmus skimmed milk was employed for the tests of coagulation and peptonization, nitrate reduction was tested in the medium recommended by Kauffmann (3). Difco gelatin was used for liquefaction, incubated at $37^{\circ} \mathrm{C}$., cooled to $20^{\circ} \mathrm{C}$. for reading and observed for one month. For $\mathrm{H}_{2} \mathrm{~S}$ production we used a medium composed of beef extract 0.75 per cent, Bacto-peptone 2 per cent, $\mathrm{NaCl} 0.5$ per cent and sodium thiosulfate 0.008 per cent, $\mathrm{pH} 7.4-7.6$. Strips of filter paper impregnated in a saturated solution of lead acetate were inserted between the tubes and the stoppers. Readings were made after 2 days incubation. For indole we employed a solution of Bacto-tryptone 1 per cent, NaCl 0.5 per cent with a pH of 7-7.2; strips of paper impregnated in oxalic acid solution were used to detect indole and when negative, Ehrlich reagent was added to the medium after 48 hours incubation. For the KCN test we used Moeller's formula with Evans' peptone 0.6 per cent instead of Orthana which was not available.

The urea test was carried out in two different ways: 1) the "slow" test, using the Christensen medium modified by Kristenson as recommended by Kauffmann but using as indicator $24 \mathrm{ml}$. per liter of a 0.04 per cent cresol red instead of 
phenol red. This change in the indicator proved to be very satisfactory as cresol red allows the detection of a higher $\mathrm{pH}$ and eliminates positive reactions with Cloaca; and 2) the "quick" test which is performed as follows: a 2 per cent solution of urea is prepared, $2 \mathrm{ml}$. of cresol red solution as above are added, and distributed in small (hemolysis) tubes containing $1 \mathrm{ml}$. each. No sterilization is needed. The tubes are inoculated with two heavy loopfuls of a 24-hour agar culture and incubated at $45-50^{\circ} \mathrm{C}$. for two hours, when positive reactions are recognized by violet-red colour. Cultures older than 24 hours are generally too alkaline and are not suitable. The best results are obtained with growth from dry agar plates. Montevideo tap water is excellent to prepare the urea solution for the test, but when distilled water is used it is convenient to add monopotasium phosphate $1 / 10,000$ to eliminate confusing weak reactions produced by some cloaca cultures. It is also recommended not to use bacteria grown on lactose agar, as under those conditions most Cloaca cultures will give a positive reaction. The arginine test was performed using the medium as recommended by Moeller but without the addition of the decarboxylase co-enzyme pyridoxal. In this way the reaction that takes place is apparently not due to decarboxylase but to the dihydrolase system as proved by the fact that without exception positive tubes gave a strong positive $\mathrm{NH}_{3}$ test. This arginine test was found useful not only to differentiate Cloaca from Klebsiella and Serratia, as shown by Moeller, but it has also the advantage that most Escherichia cultures are negative. While Moeller found that 99 per cent of his "coli" strains were positive with his decarboxylase method, we had only 13 positive in a series of 80 such cultures. For the preparation of the medium we used the local beef extract and Evans' peptone instead of Orthana brand recommended by Moeller. We have not enough experience with lysine, ornithine and glutamic acid decarboxylases to arrive at any conclusions.

\section{RESULTS}

The following tables summarize the most important characters which differentiate Klebsiella from Cloaca. As gas production from some carbohydrates is one of them, in order to avoid confusion we have tabulated anaerogenic cultures separately. We have grouped all of them according to their 
Errata: Vol.7, No.1, p.7. IMVIC signs inadvertently

omitted from Table 1 .

Table 1 . The 72 Klebsiella capsulated strains from type culture collections.

\begin{tabular}{|c|c|c|c|c|c|c|c|c|c|c|c|}
\hline \multirow[b]{2}{*}{ Group } & \multicolumn{8}{|c|}{ Gas from cellobiose } & \multicolumn{3}{|c|}{ Acid from cellobiose } \\
\hline & 1 & 2 & 3 & 4 & 5 & 6 & 7 & 8 & 1 & 3 & 4 \\
\hline IMViC & $--t+$ & $-t+t$ & -+-- & -+-+ & $=--+$ & $t-t+$ & $+t-t$ & $++t+$ & $=-++$ &.- \pm- & $-+-t$ \\
\hline No. of strains & 53 & 5 & 1 & 1 & 1 & 4 & 1 & 3 & 1 & 1 & 1 \\
\hline Dulcitol & $\begin{array}{l}\text { Gas:17 } \\
\text { Neg: } 36\end{array}$ & $\begin{array}{l}\text { Gas:1 } \\
\text { Neg: } 4\end{array}$ & Neg. & Neg. & Neg. & $\begin{array}{l}\text { Gas:1 } \\
\text { Neg: } 2 \\
\text { Ac: } 1\end{array}$ & Neg. & $\begin{array}{l}\text { Gas:2 } \\
\text { Neg:1 }\end{array}$ & Neg. & Neg. & Neg. \\
\hline Adonitol & $\begin{array}{l}\text { Neg: } 8 \\
\text { Gas: } 45 \\
\end{array}$ & Gas: 5 & Gas & Gas & Gas & Gas: 4 & Gas & Gas: 3 & Acid & Acid & Acid \\
\hline Inositol & $\begin{array}{lr}\text { Ac: } & 1 \\
\text { Ga s: } & 52 \\
\end{array}$ & Gas: 5 & Gas & Gas & Gas & Gas: 4 & Gas & Gas: 3 & Acid & Acid & Acid \\
\hline Aesculin & $\begin{array}{l}\text { Neg: } 1 \\
\text { Pos: } 52\end{array}$ & $\begin{array}{l}\text { Neg: } 1 \\
\text { Pos: } 4\end{array}$ & Pos. & Pos. & Pos. & $\begin{array}{l}\text { Neg: } 1 \\
\text { Pos: } 3\end{array}$ & Pos. & Pos: 3 & Neg. & Pos. & Pos. \\
\hline Starch & Gas:53 & Gas: 5 & Gas & Neg. & Gas & Gas: 4 & Gas & Gas:3 & Acid & Acid & Acid \\
\hline Dextrin & $\begin{array}{lr}\text { Ac: } & 2 \\
\text { Gas: } 51 \\
\end{array}$ & Gas: 5 & Gas & Acid & Gas & $\begin{array}{l}\text { Ac: } 1 \\
\text { Gas: } 3\end{array}$ & Gas & Gas:3 & Acid & Acid & Acid \\
\hline Glycerol & Gas:53 & Gas: 5 & Gas & Gas & Gas & Gas: 4 & Gas & Gas:3 & Acid & Acid & Acid \\
\hline $\begin{array}{l}\text { Urea } \\
\text { (slow) }\end{array}$ & $\begin{array}{l}\text { Pos: } 49 \\
\text { Neg: } 4 \\
\end{array}$ & $\begin{array}{l}\text { Pos:2 } \\
\text { Neg: } 3 \\
\end{array}$ & $\mathrm{Neg}$. & Neg. & Pos. & Pos: 4 & Pos. & Pos: 3 & Pos. & Neg. & Pos. \\
\hline $\begin{array}{l}\text { Urea } \\
\text { (quick) }\end{array}$ & $\begin{array}{l}\text { Pos: } 50 \\
\text { Neg: } 3\end{array}$ & $\begin{array}{l}\text { Pos: } 2 \\
\text { Neg: } 3\end{array}$ & Neg. & Neg. & Pos. & Pos: 4 & Pos. & Pos: 3 & Pos. & $\mathrm{Neg}$. & Neg. \\
\hline Gelatine & $\begin{array}{l}\text { Pos: } 3 \\
\text { Neg: } 50 \\
\end{array}$ & Neg: 5 & Pos. & Neg. & Pos. & $\begin{array}{l}\text { Pos: } 3 \\
\text { Neg: } 1\end{array}$ & Neg. & $\mathrm{Neg}: 3$ & $\mathrm{Neg}$. & $\mathrm{Neg}$. & Neg. \\
\hline $\mathrm{KCN}$ & $\begin{array}{l}\text { Pos: } 52 \\
\text { Neg: } 1 \\
\end{array}$ & Pos: 5 & Pos. & Pos. & Pos. & Pos: 4 & Pos. & Pos: 3 & Pos. & $\begin{array}{l}\text { Weak } \\
\text { Pos. }\end{array}$ & $\begin{array}{l}\text { Weak } \\
\text { Pos. }\end{array}$ \\
\hline Arginine & Neg: 53 & Neg: 5 & Neg. & Pos. & Neg. & $\mathrm{Neg}: 4$ & Neg. & Neg: 3 & Neg. & Neg. & Neg. \\
\hline
\end{tabular}



Table 1. The 72 Klebsiella capsulated strains from type culture collections.

\begin{tabular}{|c|c|c|c|c|c|c|c|c|c|c|c|}
\hline \multirow{2}{*}{ Group } & \multicolumn{8}{|c|}{ Gas from cellobiose } & \multicolumn{3}{|c|}{ Acid from cellobiose } \\
\hline & 1 & 2 & 3 & 4 & 5 & 6 & 7 & 8 & 1 & 3 & 4 \\
\hline \multicolumn{12}{|l|}{ IMViC } \\
\hline No. of strains & 53 & 5 & 1 & 1 & 1 & 4 & 1 & 3 & 1 & 1 & 1 \\
\hline Dulcitol & $\begin{array}{l}\text { Gas: } 17 \\
\text { Neg:36 }\end{array}$ & $\begin{array}{l}\text { Gas: } 1 \\
\text { Neg: } 4\end{array}$ & Neg. & Neg. & Neg . & $\begin{array}{l}\text { Gas: } 1 \\
\text { Neg: } 2 \\
\text { Ac: } 1\end{array}$ & Neg. & $\begin{array}{l}\text { Gas:2 } \\
\text { Neg:1 }\end{array}$ & Neg. & Neg. & Neg. \\
\hline Adonitol & $\begin{array}{l}\text { Neg: } 8 \\
\text { Gas: } 45 \\
\end{array}$ & Gas: 5 & Gas & Gas & Gas & Gas: 4 & Gas & Gas: 3 & Acid & Acid & Acid \\
\hline Inositol & $\begin{array}{lr}\text { Ac: } 1 \\
\text { Ga s: } 52 \\
\end{array}$ & Gas: 5 & Gas & Gas & Gas & Gas: 4 & Gas & Gas: 3 & Acid & Acid & Acid \\
\hline Aesculin & $\begin{array}{l}\text { Neg: } 1 \\
\text { Pos: } 52 \\
\end{array}$ & $\begin{array}{l}\text { Neg: } 1 \\
\text { Pos: } 4\end{array}$ & Pos. & Pos. & Pos. & $\begin{array}{l}\text { Neg: } 1 \\
\text { Pos: } 3\end{array}$ & Pos. & Pos: 3 & Neg. & Pos. & Pos. \\
\hline Starch & Gas:53 & Gas: 5 & Gas & Neg. & Gas & Gas:4 & Gas & Gas:3 & Acid & Acid & Acid \\
\hline Dextrin & $\begin{array}{l}\text { Ac: } 2 \\
\text { Gas: } 51 \\
\end{array}$ & Gas: 5 & Gas & Acid & Gas & $\begin{array}{l}\text { Ac: } 1 \\
\text { Gas: } 3\end{array}$ & Gas & Gas: 3 & Acid & Acid & Acid \\
\hline Glycerol & Gas:53 & Gas: 5 & Gas & Gas & Gas & Gas:4 & Gas & Gas:3 & Acid & Acid & Acid \\
\hline $\begin{array}{l}\text { Urea } \\
\text { (slow) }\end{array}$ & $\begin{array}{l}\text { Pos: } 49 \\
\text { Neg: } 4\end{array}$ & $\begin{array}{l}\text { Pos:2 } \\
\text { Neg: } 3 \\
\end{array}$ & Neg. & Neg. & Pos. & Pos: 4 & Pos. & Pos: 3 & Pos. & Neg. & Pos. \\
\hline $\begin{array}{l}\text { Urea } \\
\text { (quick) }\end{array}$ & $\begin{array}{l}\text { Pos: } 50 \\
\text { Neg: } 3 \\
\end{array}$ & $\begin{array}{l}\text { Pos: } 2 \\
\text { Neg: } 3\end{array}$ & Neg. & Neg. & Pos. & Pos: 4 & Pos. & Pos: 3 & Pos. & Neg. & Neg. \\
\hline Gelatine & $\begin{array}{l}\text { Pos: } 3 \\
\text { Neg: } 50 \\
\text { Poc. } 5 ?\end{array}$ & $\mathrm{Neg}: 5$ & Pos. & Neg. & Pos. & $\begin{array}{l}\text { Pos: } 3 \\
\text { Neg: } 1\end{array}$ & Neg. & Neg: 3 & $\mathrm{Neg}$. & Neg. & Neg. \\
\hline $\mathrm{KCN}$ & $\begin{array}{l}\text { Pos: } 52 \\
\text { Neg: } 1 \\
\end{array}$ & Pos: 5 & Pos. & Pos. & Pos. & Pos: 4 & Pos. & Pos: 3 & Pos. & $\begin{array}{l}\text { Weak } \\
\text { Pos. }\end{array}$ & $\begin{array}{l}\text { Weak } \\
\text { Pos. }\end{array}$ \\
\hline Arginine & $\mathrm{Neg}: 53$ & $\mathrm{Neg}: 5$ & Neg. & Pos. & Neg. & $\mathrm{Neg}: 4$ & Neg. & $\mathrm{Neg}: 3$ & Neg. & Neg. & Neg. \\
\hline
\end{tabular}


Page 8

I N TER NATIONAL BULLEIIN

IMViC reactions and it will be seen that there were 9 different combinations among Klebsiella and 5 among Cloaca. We are not suggesting that these different combinations represent subdivisions in either group, our purpose is only to show that whatever the IMViC reactions may be, the remaining properties have enough in common to place all these cultures in one group or another, and that there is a close correlation between motility and biochemical behaviour.

All the Klebsiella serotype strains from Kauffmann and Edwards fermented (with gas the aerogenic, and without it the anaerogenic), glucose, saccharose, mannitol, maltose, salicin, sorbitol, arabinose and xylose. With the exception of one culture of group 1, which produced acid only, all fermented rhamnose with gas. Four did not coagulate milk, none peptonized it, none produced $\mathrm{H}_{2} \mathrm{~S}$, two did not produce nitrites from nitrates. Lactose fermentation was delayed with serotypes $1,3,4,5$ and 6 , while all the rest promptly fermented it with abundant gas.

All locally isolated strains of Klebsiella fermented (with gas production) lactose, glucose, saccharose, mannitol, maltose, salicin, sorbitol, arabinose and xylose. One did not ferment rhamnose, another did not produce gas from it. Three did not coagulate milk, none peptonized it. All reduced nitrates to nitrites, none produced $\mathrm{H}_{2} \mathrm{~S}$, all were nonmotile.

All strains of Cloaca fermented glucose, mannitol, maltose, salicin and arabinose with production of gas; 17 fermented lactose without producing gas, 1 did not ferment it, 1 did not ferment saccharose, 9 did not ferment rhamnose, 1 fermented rhamnose but without gas; 8 did not ferment sorbitol; all save one produced gas from xylose. Two cultures did not coagulate milk, none peptonized it; 2 did not reduce nitrates to nitrites. No $\mathrm{H}_{2} \mathrm{~S}$ production was observed in any case.

Tables 1 and 2 combining the 69 aerogenic Klebsiella serotype strains and our own 286 strains, 355 in total, gave 351 which produced gas in 4 days from nonsoluble starch, or 98.9 per cent as contrasted with 4 that did not. Compared with these only one of our 177 motile cultures of Cloaca was positive, or 0.56 per cent, and even this strain, which we got from Dr. Edwards and belongs to the glycerol-and-inositolpositive, arginine-negative group mentioned below, behaved in a peculiar way. At first it produced just a small bubble of gas and only after several transfers in the same medium was there enough gas to fill the glass bell. It was then 
Errata: Vol.7, No.1, p.9.

IMVIC signs inadvertently

omitted from Table 2 .

Table 2. The 286 Klebsiella isolated strains. All produce gas from cellobiose.

\begin{tabular}{|c|c|c|c|c|c|c|c|c|}
\hline Group & 1 & 2 & 4 & 5 & 6 & 7 & 8 & 9 \\
\hline IMViC & $--t+$ & $-t+t$ & $-t-t$ & $---t$ & $t-t+$ & $t+-t$ & $t+t$ & $-+t=$ \\
\hline No. of strains & 198 & 16 & 14 & 12 & 32 & 2 & 11 & 1 \\
\hline Dulcitol & $\begin{array}{l}\text { Ac: } 1 \\
\text { Gas }: 67 \\
\text { Neg: } 130\end{array}$ & $\begin{array}{l}\text { Gas: }: 2 \\
\text { Neg: } 14\end{array}$ & $\begin{array}{l}\text { Gas: } 6 \\
\text { Neg: } 8\end{array}$ & $\begin{array}{l}\text { Gas: } 4 \\
\text { Neg: } 8\end{array}$ & $\begin{array}{l}\text { Gas: } 16 \\
\text { Neg: } 16\end{array}$ & Neg: 2 & $\begin{array}{l}\text { Gas: } 4 \\
\text { Neg: } 7\end{array}$ & Gas \\
\hline Adonitol & $\begin{array}{l}\text { Ac: } 1 \\
\text { Gas }: 157 \\
\text { Neg: } 40\end{array}$ & $\begin{array}{l}\text { Gas: } 14 \\
\text { Neg: } 2 \\
\end{array}$ & $\begin{array}{l}\text { Gas: } 13 \\
\text { Neg: } 1 \\
\end{array}$ & Gas: 12 & Gas: 32 & Gas:2 & Gas: 11 & Gas \\
\hline Inositol & $\begin{array}{l}\text { Ac: } 4 \\
\text { Gas }: 193 \\
\text { Neg: } 1\end{array}$ & Gas: 16 & $\begin{array}{l}\text { Gas: } 13 \\
\text { Neg: } 1\end{array}$ & Gas: 12 & Gas: 32 & Gas:2 & $\begin{array}{l}\text { Gas: } 10 \\
\mathrm{Ac}: 1\end{array}$ & Gas \\
\hline Aesculin & $\begin{array}{l}\text { Pos: } 197 \\
\text { Neg: } 1\end{array}$ & Pos: 16 & Pos: 14 & Pos: 12 & Pos: 32 & Pos: 2 & Pos: 11 & Pos. \\
\hline Starch & $\begin{array}{l}\text { Ac: } 2 \\
\text { Gas: } 196\end{array}$ & $\begin{array}{l}\text { Ac: } 1 \\
\text { Gas: } 15\end{array}$ & Gas: 14 & Gas: 12 & Gas: 32 & Gas:2 & Gas: 11 & Gas \\
\hline Dextrin & $\begin{array}{l}\text { Ac: } 9 \\
\text { Gas }: 189\end{array}$ & $\begin{array}{l}\text { Gas: } 15 \\
\text { Neg: } 1\end{array}$ & Gas: 14 & Gas: 12 & $\begin{array}{l}\text { Ac: } 2 \\
\text { Gas: } 29 \\
\text { Neg: } 1\end{array}$ & Gas:2 & $\begin{array}{l}\text { Ac: } 2 \\
\text { Gas: } 9\end{array}$ & Gas \\
\hline Glycerol & Gas:198 & Gas:16 & Gas:14 & Gas: 12 & Gas: 32 & Gas:2 & Gas:11 & Gas \\
\hline $\begin{array}{l}\text { Urea } \\
\text { (slow) }\end{array}$ & $\begin{array}{l}\text { Neg: } 8 \\
\text { Pos: } 190\end{array}$ & Pos: 16 & Pos: 14 & Pos: 12 & $\begin{array}{l}\text { Neg: } 3 \\
\text { Pos: } 29\end{array}$ & Pos: 2 & Pos: 11 & Pos. \\
\hline $\begin{array}{l}\text { Urea } \\
\text { (quick) }\end{array}$ & $\begin{array}{l}\text { Neg: } 3 \\
\text { Pos:195 }\end{array}$ & Pos: 16 & Pos: 14 & Pos: 12 & $\begin{array}{l}\text { Neg: } 1 \\
\text { Pos: } 31\end{array}$ & Pos: 2 & Pos: 11 & Pos. \\
\hline Gelatin & $\begin{array}{l}\text { Pos: } 29 \\
\text { Neg: } 169\end{array}$ & $\begin{array}{l}\text { Pos: } 5 \\
\text { Neg: } 11\end{array}$ & Neg: 14 & $\begin{array}{l}\text { Pos: } 2 \\
\text { Neg: } 10\end{array}$ & $\begin{array}{l}\text { Pos: } 27 \\
\text { Neg: } 5\end{array}$ & $\begin{array}{l}\text { Pos: } 1 \\
\text { Neg: } 1 \\
\end{array}$ & $\begin{array}{l}\text { Pos: } 6 \\
\text { Neg: } 5\end{array}$ & Neg. \\
\hline $\mathrm{KCN}$ & Pos: 198 & Pos: 16 & Pos: 14 & Pos: 12 & $\begin{array}{l}\text { Neg: } 1 \\
\text { Pos: } 31\end{array}$ & Pos: 2 & $\begin{array}{l}\text { Neg: } 1 \\
\text { Pos: } 10\end{array}$ & Pos. \\
\hline Arginine & $\begin{array}{l}\text { Pos: } 4 \\
\text { Neg: } 194\end{array}$ & Neg: 16 & Neg: 14 & Neg: 12 & Neg: 32 & Neg: 2 & Neg: 11 & Neg. \\
\hline Capsule & $\begin{array}{l}\text { Pos: } 166 \\
\text { Neg: } 32\end{array}$ & $\begin{array}{l}\text { Pos: } 14 \\
\text { Neg: } 2\end{array}$ & $\begin{array}{l}\text { Pos: } 12 \\
\text { Neg: } 2\end{array}$ & $\begin{array}{l}\text { Pos: } 10 \\
\text { Neg: } 2\end{array}$ & $\begin{array}{l}\text { Pos: } 25 \\
\text { Neg: } 7\end{array}$ & $\begin{array}{l}\text { Pos: } 1 \\
\text { Neg: } 1\end{array}$ & $\begin{array}{l}\text { Pos: } 8 \\
\text { Neg: } 3\end{array}$ & Neg. \\
\hline
\end{tabular}



Table 2. The 286 Klebslella 1solated strains. All produce gas fran celloblose.

\begin{tabular}{|c|c|c|c|c|c|c|c|c|}
\hline \multirow{2}{*}{\multicolumn{9}{|c|}{$\begin{array}{l}\text { Group } \\
\text { IMViC }\end{array}$}} \\
\hline & & & & & & & & \\
\hline No. of strains & 198 & 16 & 14 & 12 & 32 & 2 & 11 & 1 \\
\hline Dulcitol & $\begin{array}{l}\text { Ac: } 1 \\
\text { Gas: } 67 \\
\text { Neg: } 130\end{array}$ & $\begin{array}{l}\text { Gas: } 2 \\
\text { Neg: } 14\end{array}$ & $\begin{array}{l}\text { Gas: } 6 \\
\text { Neg: } 8\end{array}$ & $\begin{array}{l}\text { Gas: } 4 \\
\text { Neq: } 8 \\
\end{array}$ & $\begin{array}{l}\text { Gas: } 16 \\
\text { Neg: } 16 \\
\end{array}$ & Neg:2 & $\begin{array}{l}\text { Gas: } 4 \\
\text { Neg: } 7\end{array}$ & Gas \\
\hline Adonitol & $\begin{array}{l}\text { Ac: } 1 \\
\text { Gas: } 157 \\
\text { Neg: } 40\end{array}$ & $\begin{array}{l}\text { Gas: } 14 \\
\text { Neg: } 2 \\
\end{array}$ & $\begin{array}{l}\text { Gas: } 13 \\
\text { Neg: } 1 \\
\end{array}$ & Gas: 12 & Gas: 32 & Gas:2 & Gas:11 & Gas \\
\hline Inositol & $\begin{array}{l}\text { Ac: } 4 \\
\text { Gas: } 193 \\
\text { Neg: } 1 \\
\end{array}$ & Gas: 16 & $\begin{array}{l}\text { Gas: } 13 \\
\text { Neg: } 1\end{array}$ & Gas: 12 & Gas: 32 & Gas:2 & $\begin{array}{l}\text { Gas: } 10 \\
\text { Ac: } 1 \\
\end{array}$ & Gas \\
\hline Aesculin & $\begin{array}{l}\text { Pos: } 197 \\
\text { Neg: } 1\end{array}$ & Pos: 16 & Pos:14 & Pos: 12 & Pos: 32 & Pos: 2 & Pos: 11 & Pos. \\
\hline Starch & $\begin{array}{l}\text { Ac: } 2 \\
\text { Gas: } 196\end{array}$ & $\begin{array}{l}\text { Ac: } 1 \\
\text { Gas: } 15\end{array}$ & Gas: 14 & Gas:12 & Gas: 32 & Gas: 2 & Gas:11 & Gas \\
\hline Dextrin & $\begin{array}{l}\text { Ac: } 9 \\
\text { Gas: } 189\end{array}$ & $\begin{array}{l}\text { Gas: } 15 \\
\text { Neg: } 1\end{array}$ & Gas:14 & Gas: 12 & $\begin{array}{l}\text { Ac: } 2 \\
\text { Gas: } 29 \\
\text { Neg: } 1\end{array}$ & Gas: 2 & $\begin{array}{l}\text { Ac: } 2 \\
\text { Gas: } 9\end{array}$ & Gas \\
\hline Glycerol & Gas:198 & Gas: 16 & Gas: 14 & Gas: 12 & Gas: 32 & Gas:2 & Gas:11 & Gas \\
\hline $\begin{array}{l}\text { Urea } \\
\text { (slow) }\end{array}$ & $\begin{array}{l}\text { Neg: } 8 \\
\text { Pos:190 }\end{array}$ & Pos: 16 & Pos: 14 & Pos: 12 & $\begin{array}{l}\text { Neg: } 3 \\
\text { Pos:29 }\end{array}$ & Pos:2 & Pos: 11 & Pos. \\
\hline $\begin{array}{l}\text { Urea } \\
\text { (quick) }\end{array}$ & $\begin{array}{l}\text { Neg: } 3 \\
\text { Pos: } 195\end{array}$ & Pos: 16 & Pos: 14 & Pos: 12 & $\begin{array}{l}\text { Neg: } 1 \\
\text { Pos: } 31\end{array}$ & Pos: 2 & Pos: 11 & Pos. \\
\hline Gelatin & $\begin{array}{l}\text { Pos: } 29 \\
\text { Neg: } 169\end{array}$ & $\begin{array}{l}\text { Pos: } 5 \\
\text { Neg: } 11 \\
\end{array}$ & Neg: 14 & $\begin{array}{l}\text { Pos: } 2 \\
\text { Neg: } 10\end{array}$ & $\begin{array}{l}\text { Pos: } 27 \\
\text { Neg: } 5\end{array}$ & $\begin{array}{l}\text { Pos:1 } \\
\text { Neg:1 }\end{array}$ & $\begin{array}{l}\text { Pos: } 6 \\
\text { Neg: } 5 \\
\end{array}$ & Neg. \\
\hline $\mathrm{KCN}$ & Pos: 198 & Pos: 16 & Pos: 14 & Pos:12 & $\begin{array}{l}\text { Neg: } 1 \\
\text { Pos: } 31\end{array}$ & Pos: 2 & $\begin{array}{l}\text { Neg: } 1 \\
\text { Pos: } 10\end{array}$ & Pos. \\
\hline Arginine & $\begin{array}{l}\text { Pos: } 4 \\
\text { Neg: } 194\end{array}$ & Neg: 16 & Neg:14 & Neg:12 & Neg: 32 & Neg: 2 & Neg: 11 & Neq. \\
\hline Capsule & $\begin{array}{l}\text { Pos: } 166 \\
\text { Neg: } 32\end{array}$ & $\begin{array}{l}\text { Pos:14 } \\
\text { Neg: } 2\end{array}$ & $\begin{array}{l}\text { Pos: } 12 \\
\text { Neg: } 2\end{array}$ & $\begin{array}{l}\text { Pos: } 10 \\
\text { Neg: } 2\end{array}$ & $\begin{array}{l}\text { Pos: } 25 \\
\text { Neg: } 7\end{array}$ & $\begin{array}{l}\text { Pos:1 } \\
\text { Neg: } 1\end{array}$ & $\begin{array}{l}\text { Pos: } 8 \\
\text { Neg: } 3\end{array}$ & Neg. \\
\hline
\end{tabular}


Table 3. 202 cloaca strains. All gas from cellobiose.

\begin{tabular}{|c|c|c|c|c|c|c|c|c|c|}
\hline \multirow{3}{*}{ Group } & \multicolumn{5}{|c|}{177 mot 11e forms } & \multicolumn{4}{|c|}{25 "O" forms } \\
\hline & 1 & 2 & 4 & 5 & 10 & 1 & 2 & 4 & 5 \\
\hline & & & & & & & & & \\
\hline No. of strains & 169 & 1 & 5 & 1 & 1 & 16 & 7 & 1 & 1 \\
\hline Dulcitol & $\begin{array}{l}\text { Ac: } 1 \\
\text { Gas: } 25 \\
\text { Neg: } 143 \\
\end{array}$ & Neg. & $\begin{array}{l}\text { Gas: } 2 \\
\text { Neg: } 3 \\
\end{array}$ & Gas & Neg. & $\begin{array}{l}\text { Gas: } 1 \\
\text { Neg: } 15\end{array}$ & $\begin{array}{l}\text { Ac: } 1 \\
\text { Neg: } 6\end{array}$ & Neg. & Neg. \\
\hline Adonitol & $\begin{array}{l}\text { Ac: } 1 \\
\text { Gas: } 63 \\
\text { Neg: } 105\end{array}$ & Neg. & $\begin{array}{l}\text { Neg: } 4 \\
\text { Gas:1 }\end{array}$ & Neg. & Neg. & $\begin{array}{l}\text { Ac: } 2 \\
\text { Neg: } 12 \\
\text { Gas: } 2 \\
\end{array}$ & Neg: 7 & Neg. & Neg. \\
\hline Inositol & $\begin{array}{l}\text { Gas: } 27 \\
\text { Ac: } 71 \\
\text { Neg: } 71 \\
\end{array}$ & Ac1d & $\begin{array}{l}\text { Ac: } 2 \\
\text { Neg: } 3\end{array}$ & Acid & Gas & $\begin{array}{l}\text { Gas: } 3 \\
\text { Ac: } 3 \\
\text { Neg: } 10\end{array}$ & $\begin{array}{l}\text { Ac: } 1 \\
\text { Neg: } 6\end{array}$ & Acid & Acid \\
\hline Aesculin & $\begin{array}{l}\text { Pos: } 55 \\
\text { Neg: } 114 \\
\text { Gas: } 1\end{array}$ & Neg. & $\begin{array}{l}\text { Pos: } 1 \\
\text { Neg: } 4\end{array}$ & Pos. & Pos. & $\begin{array}{l}\text { Pos: } 12 \\
\text { Neg: } 4\end{array}$ & Pos:7 & Pos. & Neg. \\
\hline Starch & $\begin{array}{l}\text { Ac: }: 49 \\
\text { Neg: } 119 \\
\end{array}$ & Neg. & $\begin{array}{l}\text { Ac: } 1 \\
\text { Neg: } 4\end{array}$ & Neg. & Acid & $\begin{array}{l}\text { Ac: } 2 \\
\text { Neg: } 14\end{array}$ & Neg: 7 & Neg. & Acid \\
\hline Dextrin & $\begin{array}{l}\text { Gas: } 24 \\
\text { Ac: } 99 \\
\text { Neg: } 46\end{array}$ & Gas & $\begin{array}{l}\text { Gas:3 } \\
\text { Neg:2 }\end{array}$ & Neg. & Acid & $\begin{array}{l}\text { Gas: } 1 \\
\text { Ac: } 5 \\
\text { Neg: } 10 \\
\end{array}$ & $\begin{array}{l}\text { Ac: } 3 \\
\text { Neg: } 4\end{array}$ & Acid & Acid \\
\hline Glycerol & $\begin{array}{l}\text { Gas: } 27 \\
\text { Ac: } 62 \\
\text { Neg: } 80 \\
\end{array}$ & Neg. & $\begin{array}{l}\text { Ac: } 3 \\
\text { Neg:2 }\end{array}$ & Acid & Gas & $\begin{array}{l}\text { Gas: } 1 \\
\text { Ac: } 11 \\
\text { Neg: } 4\end{array}$ & $A c: 7$ & Acid & Acid \\
\hline Urea (slow) & Neg:169 & Neg. & Neg: 5 & Neg. & Neg. & Neg: 16 & Neg: 7 & Neg. & Neg. \\
\hline Urea (quick) & Neg:169 & Neg. & $\mathrm{Neg}: 5$ & Neg. & Neg. & Neg: 16 & Neg:7 & Neg. & Neg. \\
\hline Gelatin & $\begin{array}{l}\text { Pos: } 154 \\
\text { Neg: } 15\end{array}$ & Pos. & $\begin{array}{l}\text { Pos: } 4 \\
\text { Neg: } 1 \\
\end{array}$ & Pos. & Neg. & $\begin{array}{l}\text { Pos: } 14 \\
\text { Neg: } 2\end{array}$ & $\begin{array}{l}\text { Pos: } 6 \\
\text { Neg: } 1\end{array}$ & Pos. & Pos. \\
\hline $\mathrm{KCN}$ & $\begin{array}{l}\text { Neg: } 2 \\
\text { Pos: } 167\end{array}$ & Pos. & Pos: 5 & Pos. & Pos. & Pos: 16 & Pos: 7 & Pos. & Pos. \\
\hline Arginine & $\begin{array}{l}\text { Pos: } 146 \\
\text { Neg: } 23\end{array}$ & Pos. & $\begin{array}{l}\text { Pos: } 4 \\
\text { Neg: } 1\end{array}$ & Pos. & Neg. & $\begin{array}{l}\text { Pos: } 14 \\
\text { Neg:2 }\end{array}$ & $\begin{array}{l}\text { Pos: } 4 \\
\text { Neg: } 3\end{array}$ & Neg. & Pos. \\
\hline Capsule & $\begin{array}{l}\text { Pos: } 15 \\
\text { Neg: } 154\end{array}$ & Pos. & Neg: 5 & Neg. & Neg. & $\begin{array}{l}\text { Pos: } 1 \\
\text { Neg: } 15 \\
\end{array}$ & $\begin{array}{l}\text { Pos: } 1 \\
\text { Neg: } 6\end{array}$ & Neg. & Neg. \\
\hline
\end{tabular}




\begin{tabular}{|c|c|c|c|c|c|c|c|c|c|}
\hline & & 17 & tile fo & & & & 25 "O" & forms & \\
\hline Group & 1 & 2 & 4 & 5 & 10 & 1 & 2 & 4 & 5 \\
\hline IMVIC & --++ & $-t+t$ & $-+-t$ & ---+ & --+- & $--t+$ & $-t+t$ & $-t-t$ & $---t$ \\
\hline No. of strains & 169 & 1 & 5 & 1 & 1 & 16 & 7 & 1 & 1 \\
\hline Dulcitol & $\begin{array}{l}\text { Ac: } 1 \\
\text { Gas: } 25 \\
\text { Neg: } 143\end{array}$ & Neg. & $\begin{array}{l}\text { Gas: } 2 \\
\text { Neg: } 3\end{array}$ & Gas & Neg. & $\begin{array}{l}\text { Gas: } 1 \\
\text { Neg: } 15\end{array}$ & $\begin{array}{l}\text { Ac: } 1 \\
\text { Neg: } 6\end{array}$ & Neg. & Neg. \\
\hline Adonitol & $\begin{array}{l}\text { Ac: } 1 \\
\text { Gas: } 63 \\
\text { Neg: } 105\end{array}$ & Neg. & $\begin{array}{l}\text { Neg: } 4 \\
\text { Gas:1 }\end{array}$ & Neg. & Neg. & $\begin{array}{l}\text { Ac: } 2 \\
\text { Neg: } 12 \\
\text { Gas: } 2\end{array}$ & Neg: 7 & Neg. & Neg. \\
\hline Inositol & $\begin{array}{l}\text { Ga s: } 27 \\
\text { Ac }: 71 \\
\text { Neg }: 71\end{array}$ & Ac1d & $\begin{array}{l}\text { Ac: } 2 \\
\text { Neg: } 3\end{array}$ & Acid & Gas & $\begin{array}{l}\text { Gas: } 3 \\
\text { Ac: } 3 \\
\text { Neg: } 10\end{array}$ & $\begin{array}{l}\text { Ac: } 1 \\
\text { Neg: } 6\end{array}$ & Acid & Acid \\
\hline Aesculin & $\begin{array}{l}\text { Pos: } 55 \\
\text { Neg: } 114\end{array}$ & $\mathrm{Neg}$. & $\begin{array}{l}\text { Pos: } 1 \\
\text { Neg: } 4\end{array}$ & Pos. & Pos. & $\begin{array}{l}\text { Pos: } 12 \\
\text { Neg: } 4\end{array}$ & Pos: 7 & Pos. & Neg. \\
\hline Starch & $\begin{array}{l}\text { Gas: } 1 \\
\text { Ac: } 49 \\
\text { Neg: } 119\end{array}$ & Neg. & $\begin{array}{l}\text { Ac: } 1 \\
\text { Neg: } 4 \\
\end{array}$ & Neg. & Acid & $\begin{array}{l}\text { Ac: } 2 \\
\text { Neg: } 14\end{array}$ & Neg: 7 & Neg. & Acid \\
\hline Dextrin & $\begin{array}{l}\text { Gas: } 24 \\
\text { Ac: } 99 \\
\text { Neg: } 46\end{array}$ & Gas & $\begin{array}{l}\text { Gas: } 3 \\
\text { Neg:2 }\end{array}$ & Neg. & Acid & $\begin{array}{l}\text { Gas: } 1 \\
\text { Ac: } 5 \\
\text { Neg: } 10\end{array}$ & $\begin{array}{l}\text { Ac: }: 3 \\
\text { Neg: } 4\end{array}$ & Acid & Acid \\
\hline Glycerol & $\begin{array}{l}\text { Gas: } 27 \\
\text { Ac: } 62 \\
\text { Neg: } 80\end{array}$ & Neg. & $\begin{array}{l}\text { Ac: } 3 \\
\text { Neg: } 2\end{array}$ & Acid & Gas & $\begin{array}{l}\text { Gas: } 1 \\
\text { Ac: } 11 \\
\mathrm{Neg}: 4\end{array}$ & Ac: $: 7$ & Acid & Acid \\
\hline Urea (slow) & Neg:169 & $\mathrm{Neg}$. & Neg: 5 & $\mathrm{Neg}$. & Neg. & Neg: 16 & Neg: 7 & $\mathrm{Neg}$. & Neg. \\
\hline Urea (quick) & Neg:169 & Neg. & $\mathrm{Neg}: 5$ & Neg. & Neg. & Neg:16 & Neg: 7 & Neg. & Neg. \\
\hline Gelatin & $\begin{array}{l}\text { Pos: } 154 \\
\text { Neg: } 15\end{array}$ & Pos. & $\begin{array}{l}\text { Pos: } 4 \\
\text { Neg: } 1\end{array}$ & Pos. & $\mathrm{Neg}$. & $\begin{array}{l}\text { Pos: } 14 \\
\text { Neg: } 2\end{array}$ & $\begin{array}{l}\text { Pos: } 6 \\
\text { Neg: } 1\end{array}$ & Pos. & Pos. \\
\hline $\mathrm{KCN}$ & $\begin{array}{l}\text { Neg: } 2 \\
\text { Pos: } 167\end{array}$ & Pos. & Pos: 5 & Pos. & Pos. & Pos: 16 & Pos: 7 & Pos. & Pos. \\
\hline Arginine & $\begin{array}{l}\text { Pos: } 146 \\
\text { Neg: } 23\end{array}$ & Pos. & $\begin{array}{l}\text { Pos: } 4 \\
\text { Neg: } 1\end{array}$ & Pos. & Neg. & $\begin{array}{l}\text { Pos: } 14 \\
\text { Neg: } 2\end{array}$ & $\begin{array}{l}\text { Pos: } 4 \\
\text { Neg: } 3\end{array}$ & Neg. & Pos. \\
\hline Capsule & $\begin{array}{l}\text { Pos: } 15 \\
\text { Neg: } 154\end{array}$ & Pos. & Neg: 5 & $\mathrm{Neg}$. & Neg. & $\begin{array}{l}\text { Pos: } 1 \\
\text { Neg: } 15\end{array}$ & $\begin{array}{l}\text { Pos: } 1 \\
\text { Neg: } 6\end{array}$ & $\mathrm{Neg}$. & Neg. \\
\hline
\end{tabular}



plated, and we got positive and negative colonies. When both of these were replated the positive ones went on producing numerous negative variants, while the negative ones only produced a few or no positive colonies. It appears that gas production from starch is not a fixed character of this strain.

The tables also show that for the purpose of classification, gas production from starch, not acid productions alone, is of significance, as many motile cloaca strains did produce acid. For comparison we have included in the tables our results, with dextrin, which proved not to be as useful as starch. If we consider gas production from dextrin, 336 out of 355 Klebsiella cultures or 94 per cent were positive, but so were 28 of our 177 Cloaca strains, or 15 per cent; if we consider acid production we have only 2 negative Klebsiellae, or more than 99 per cent positive, but there were also 128 of 177 Cloacae positive, or 72 per cent. Therefore all we can say about dextrin is that in our opinion a culture in the family Enterobacteriaceae not fermenting it almost certainly is not Klebsiella.

When using the "slow test" for urea fermentation, we had in all 337 or 94 per cent of Klebsiella strains positive, and 344 or 96 per cent with our "quick test." No motile Cloaca culture was positive in either. Although the quick test gave better results we do not consider it as advisable for routine, as it involves more work; we reserve it for doubtful cases or when quick diagnosis is necessary.

In our opinion gelatin liquefaction does not deserve the importance that is attributed to it as one of the fundamental characters, if not the most important, in the differentiation of Klebsiella and Cloaca. It is true that no more than 17 or 9.6 per cent of our motile cultures were negative, but on the other hand we had 78 or 22 per cent positive nonmotile cultures which exhibited all the other characters of the Klebsiella group and which could not logically be put with the motile forms. It is interesting to note that IMViC group 6 of Klebsiella $(+-++)$ was the one containing the highest percentage of gelatin liquefiers, as 3 out of 4 serotype strains and 27 of 32 of our own were liquefiers, in total 30 positive in 36 , or 83 per cent. We could not observe any significant difference in the speed of liquefaction although in general Cloaca cultures were more active than Klebsiella; the shortest period was 3 days, observed with one culture of each group, but generally liquefaction was observable between 10 and 20 days. 
Dulcitol was fermented by 123 out of 358 or 34 per cent of Klebsiella cultures and by 29 of 177 or 16 per cent of Cloaca strains, figures which are more in agreement with Moeller and with Edwards and Fife than with Brooke. As for adonitol we had 307 or 85 per cent positive Klebsiella and 65 or 3 per cent positive Cloaca. Salicin was fermented by all cultures of the two groups, even if a little slower by Cloaca; therefore in our experience these three carbohydrates are not useful for purposes of classification. In agreement with both Brooke and Moeller we found that 356 of our 358 Klebsiella cultures fermented inositol, almost 100 per cent, but 103 or 58 per cent motile cultures were also positive, in contrast with Brooke's results, and confirming the figures of Moeller and of Edwards and Fife. However, if gas production is considered, it will be seen in our tables that only 6 of the aerogenic Klebsiella cultures were negative, 98.4 per cent positive) whereas 28 out of 177 motile Cloaca strains were positive, or 15 per cent. As for arginine 152 or 85 per cent of 177 Cloaca cultures were positive against 5 positive Klebslella in 358 , or 1.4 per cent.

All aerogenic Klebsiella cultures produced gas from glycerol in 4 days, and in contrast only 28 or 15 per cent of Cloaca strains were gas-positive, which means that if this character alone had been considered, 85 per cent of them would have been rightly classifled. We think therefore that the 3 characters, gas production from inositol and glycerol, and the arginine test are very useful, and when considering cultures isolated in Montevideo, they were especially useful as proved by the following analysis of our figures. From our $28 \mathrm{Cloaca}$ cultures producing gas from glycerol, 23 also produced it from inositol and were arginine-negative. The remaining 5 behaved normally with respect to arginine, that is to say, were positive, and of these only one was also positive for gas in inositol, while 4 were negative. Two arginine negative cultures did not produce gas from either inositol or glycerol. It follows, therefore, that there is a group of Cloaca cultures, which for brevity we will call "type B", which combines the three unusual characters of producing gas from inositol and glycerol and being arginine-negative, and the correlation of these, although not perfect, is high. of these 23 "type $B$ " cultures, 18 were among those we recelved from Dr. Edwards, and as mentioned before, we have included 43 of his strains in Table 3. This means that 41 per cent of them belonged here, while among the Montevideo 
isolated cultures we found only 5 or 3.6 per cent. A possible explanation for the very different results obtained by Brooke working in Denmark and Edwards and Fife in the U.S.A. on gas production from glycerol by bacterla of the Cloaca group is that it may be due to different geographical distribution or source of isolation of the cultures tested by these authors. In any case the three tests were of considerable value to us as we had only 7.4 per cent glycerol or inositol gas-positive and less than 5 per cent arginine-negative locally isolated Cloaca cultures. Inulin was also tested with all cultures, but it proved of no interest, and we have only to mention that some klebsiellae fermented it, especially indole-positive strains.

The usefulness of the aesculin test is restricted to a few cases, but even so, this test may be of importance in confirming a doubtful diagnosis; only 1 of 286 Klebsiella cultures isolated in Montevideo was negative in contrast to 70 per cent of motile Cloaca strains. It will be observed though that there were 4 negative strains among the $72 \mathrm{Kleb}-$ siella serotypes. Capsules were observed in 82 per cent of our Montevideo Klebsiella cultures and (confirming Edwards and $\mathrm{Fife}$ ) also in 9 per cent of those of motile Cloaca strains. All Klebsiella cultures, including our local strains and all of those of Cloaca, promptly fermented cellobiose and only 5 in a total of 569 tested cultures, or 0.8 per cent were $\mathrm{KCN}-$ negative. No indole-positive, motile or nonmotile Cloaca strains were found. We had 5 motile strains resembling the group, two of them with IMViC ++-+ and $3++--$, which promptly fermented cellobiose with gas, some arginine or KCNpositive, but we think they are "intermediates" more closely related to Escherichia than to Cloaca on the basis of the other biochemical tests.

It may be interesting to mention the unusual behaviour of two strains not included in the tables. One of them, a nonmotile - - + , capsulated, non-gelatin-liquefylng, typical Klebsiella culture produced gas only in starch and dextrin, and transfers from these media to all other carbohydrates were constantly gas-negative; another, also a nonmotile, capsulated -+-+ , urea-negative and arginine-negative culture, produced gas from adonitol, inositol, salicin, cellobiose, glycerol, starch, dextrin and other carbohydrates, but not from glucose, saccharose or mannitol, and all transfers from gas-positive tubes to these three carbohydrates were negative for gas. We think this strain should be considered as a ureanegative Klebsiella. 


\section{DISCUSSION}

Although we are not prepared to arrive at final conclusions because the number of tested cultures is too small and also because geographical distribution may be of importance, as illustrated by the small number of "B type" Cloaca cultures isolated in Montevideo, we would like to emphasize some points which seem to us of practical value for routine work.

In our opinion cellobiose and $\mathrm{KCN}$ tests should be adopted by laboratories engaged in work on the Enterobacteriaceae. The first we found specially useful to differentiate cultures of anaerogenic, nonpigment-producing Serratia from Cloaca, a problem which frequently arises when dealing with strains isolated from human stools, bile, sputum or wounds. It is true that the first are arginine- and rhamnose-negative, but so are some strains of Cloaca; usually Serratia strains promptly liquefy gelatin (in one or two days) and peptonize milk, but when they do not cellobiose is important, as it is either not fermented by the group or very slowly. We have to add though, that among the aerogenic species of the group there are some, including at least some strains of $\underline{S}$. marcescens, which promptly ferment cellobiose and several other carbohydrates with abundant gas, and that as their IMViC reactions are - -++ and they are $\mathrm{KCN}$-positive, they could be very easily mistaken for strains of Cloaca if they did not produce pigment. We can confirm Moeller's statement about the usefulness of the KCN test, for differentiation of Escherichia coli from Klebsiella and Cloaca.

But even with all the tests we used we wonder if some of our strains really belong to either of the two groups. For instance, it could be that some motile cultures tentatively included in IMV1C group 4 are more closely related to the Bethesda-Freundii group than to Cloaca inasmuch as lossvariant, non- $\mathrm{H}_{2} \mathrm{~S}$ producing strains of the first group could be very difficult to differentiate from Cloaca.

Since biochemical behaviour is accepted as the basis for defining the Enterobacteriaceae, we think that our results define the Klebsiella and Cloaca groups as follows:

Klebsiella. Nonmotile, promptly fermenting inositol and glycerol with gas, urea-positive, producing gas in 4 days from nonsoluble starch, arginine-negative and with the exception of some particular IMViC combinations, usually not liquefying gelatin. 
Cloaca. Motile, usually not producing gas from inositol and glycerol (with the exception of the "B type"), ureanegative, generally arginine-positive and gelatin-positive and when fermenting nonsoluble starch not producing gas from it.

To these definitions should be added cellobiose fermentation and the KCN test, and mention should be made of the different IMViC combinations already known, as we do not see the point in calling so many strains "aberrant" that do not behave like group 1. It follows therefore that the 25 nonmotile cultures included in Table 3 have to be considered as Cloaca "O" forms since there is no character to differentlate them from the motile cultures in the same IMViC groups, although of course we do not know if they are variants derived from motile bacteria.

There is another consequence to which we would call attention, namely a culture in Table 1 in IMViC group $4(-+-+)$; the "Ozena" capsular antigen 5 culture, Dr. Kauffmann's 5051. For the same reasons this particular strain has to be considered as an "O" form of Cloaca, since it is urea-negative, does not ferment starch and is positive in arginine, three of the most important characters of the group. This observation induced us to test more strains of this and of the 3 , 4 and 6 serotypes, the two first included in Table 1 in groups 3 and 4 respectively of anaerogenic serotype cultures and the last in group 3 of the aerogenic ones, as none of them could be satisfactorlly classifled. We observed also that all of these and type 1 as mentioned before were the only Klebsiella strains showing delayed lactose fermentation, a character not infrequent among Cloaca cultures but which we have as yet never seen in other klebslellae. For the purpose we employed 40 nonmotile capsulated strains serologlcally typed by Dr. Edwards which he kindly sent to us, all of them aerogenic, promptly fermenting cellobiose with gas, and aesculin-positive. We added two cultures of $\underline{K}$. rhinoscleromat1s which we obtained from the A.T.C.C., strains 6908 and 9436. The results were as follows:

1) Ten cultures of serological type 3, promptly fermented lactose and glycerol with gas and were arginine-negative. They could be divided into four groups.

a) Three cultures with IMVIC reactions,$-+-+ \mathrm{KCN}-$, urea + , producing gas from inositol and starch, gelatin - 
(2 strains), + (1 strain);

b) three with IMViC reactions - - + $+\mathrm{KCN}+$, urea + , producing gas from starch, gas from inositol (2), no gas from inositol (1), gelatin $+(2),-(1)$;

c) three with IMVIC reactions,$+-++ \mathrm{KCN}+$, gelatin + , gas from inositol and starch, urea $+(2),-(1)$; and d) one with IMViC reactions,$++-+ \mathrm{KCN}+$, urea + , gelatin + , gas from inositol and starch.

The two cultures from the A.T.C.C. were anaerogenic, their IMViC reactions were - + - , they were lactose -, urea -, starch -, inositol + , glycerol + , aesculin + , gelatin -, arginine -. One of them grew weakly in $\mathrm{KCN}$ while the other did not grow.

The culture of Dr. Kauffmann's K. rhinoscleromatis 5046 is very similar to these two. Like them its IMVIC is - + - , It is glycerol and inositol + , urea - and arginine -, aesculin + , and grows poorly in KCN. It differs though in fermenting lactose and starch slowly, but as it is anaerogenic, this and other carbohydrate tests are of Iittle use for classification.

In our opinion the two strains coming from the A.T.C.C. have to be considered as members of Cloaca or at least more closely related to this group than to Klebsiella as they are urea - and starch -. The systematic position of the type culture (originally NCTC 5046) seems to us rather doubtful and needs closer investigation. K. rhinoscleromatis is considered generally not only as a capsular serologic type but as a species recognizable by its blochemical characters. Among these Dr. Moeller says that all four decarboxylase tests are negative, and this is the only type which behaves in this way. In his paper he only mentions having tested one culture; on the other hand we have among our strains of Cloaca one mot1le and two " $O$ " forms also negative in the four tests, which seems rather suggestive. Whatever the position of $\mathrm{K}$. rhinoscleromat1s, it 15 evident that the 10 strains of Dr. Edwards, although belonging to the same antigenic capsular group, are very different from it, and in our opinion have to be classifled in Klebsiella.

2) Nineteen cultures of serotype 4 were divided into three groups.

a) Sixteen w1th IMVIC reactions -++ which behaved as Klebsiella. They were all gas positive in inositol, 
B A C T E R IOLOG ICA L NOMENCLATURE A N D T A X ONOMY

glycerol and starch, promptly fermented lactose and split urea, and were arginine -; one grew poorly in $\mathrm{KCN}$ while the remaining $15 \mathrm{grew}$ well, and 4 liquefied gelatin;

b) two strains with IMViC reactions - + - +, urea -, starch -, and gelatin -, fermented inositol with gas and differed in that one was arginine,$+ \mathrm{KCN}$ - and fermented glycerol with gas, while the second was $\mathrm{KCN}+$, arginine and glycerol -;

c) one culture with IMVIC reaction - - + , fermented inositol and glycerol with gas, was arginine -, $\mathrm{KCN}-$, urea -, starch - and gelatin -.

All three strains fermented lactose in 4 to 6 days, and we think they belong in Cloaca.

The serotype culture, Dr. Kauffmann's "Ozena" 5050 is another anaerogenic culture whose IMVIC is - +-+ , arginine and gelatin -, grows weakly in KCN and ferments starch; as for urea, the slow test was positive and the quick test was negative. We do not think it possible to give an opinion about its taxonomic position, although it seems to be close to the two strains of Cloaca with the same IMViC mentioned in b).

3) Six cultures of serological type 5, all gelatin-negative and promptly fermenting lactose, divided into three groups.

a) One strain with IMViC - + - - did not ferment starch or dextrin, fermented glycerol without gas, produced gas from inositol, was urea -, arginine + and weakly positive in $\mathrm{KCN}$;

b) three strains with IMViC -+-+ , fermented inositol with gas, glycerol with acid only, two did not ferment starch, one produced acid only, all three fermented dextrin without gas, were urea - and grew poorly in $\mathrm{KCN}$, one was arginine + and two were - ;

c) two - + + cultures with typical Klebsiella biochemical behaviour; gas production from inositol + , glycerol + and starch + , urea,$+ \mathrm{KCN}+$ and arginine - .

As stated before in our opinion the serotype culture 5050 belongs to the Cloaca group and is very similar to the three strains mentioned in b) which differed from it only in that they did not produce gas from glycerol and that two of them were arginine - . As for the last two strains they completely differ from the serotype culture. 
4) One culture of type 6, and 5 others serologically related, were all typical - - + strains of Klebslella, splitting urea, arginine -, producing gas from starch, glycerol and inositol, and $\mathrm{KCN}+$. One liquefied gelatin. The systematic position of Dr. Kauffmann's F 5052, included in IMViC group 3, is not quite clear as it does not ferment urea and liquefies gelatin but produces gas from starch, glycerol and inositol, and is arginine -. We think it is probably an aberrant Klebsiella. All the 6 cultures tested differed from it.

When we compare the behaviour of all these strains with the respective serotype cultures, it is obvious that there is no correlation between capsular antigens and biochemical characters, a fact that was to be expected since Edwards has pointed out that some motile strains share the capsular antigens of Klebsiella, and 1 is is known that they can even be found in the Escherichia group. Therefore for purposes of classification capsular typing cannot be used alone.

As was also to be expected there are some "Intermediate" nonmotile cultures whose position cannot be satisfactorily decided as they share some characters of Klebsiella and of Cloaca. They do not seem to be of frequent occurrence since we did not find any among our own or Dr. Edwards' cultures, but only among serotype strains which constitute a highly selected material. Although a better knowledge of both groups is needed to solve the problem that these strains pose to the taxonomist, it is our opinion that the above mentioned tests should prove to be useful for routine work and in most cases sufficlent to arrive at a correct classification.

As for the name to be given to the motile group, we feel inclined to agree with $\mathrm{Dr}$. Edwards' suggestion that Aerobacter Cloaca should be adopted, with of course, the redefinition of that genus.

\section{CONCLUSIONS}

1) We suggest that the distinguishing characters of both groups, Klebslella and Cloaca, should include celloblose fermentation and $\mathrm{KCN}$ tests.

2) Separation of the two groups should be based on motility, urea hydrolysis, gas production from nonsoluble starch, inositol and glycerol, the arginine dihydrolase test and gelatin liquefaction, with mention of the frequency with 
which the +-++ IMViC group of Klebsiella liquefy it. Reference should be made to the Cloaca "B type". The definition of both groups should also include the different known combinations of IMViC.

3) There is no correlation between capsular antigens and biochemical behaviour of the two groups so that serological typing cannot be used alone for identification of nonmotile cultures. Among 40 tested cultures of types 3, 4, 5 and 6 , not one was exactly like the serotype culture, and there were also Klebsiella and Cloaca "O" forms.

4) The cultures of rhinoscleroma and ozena types 4 and 6 cannot be placed in Klebsiella or Cloaca without further investigation, as they seem to be "intermediates" between the two groups. In our opinion, the serotype of type 5 is an "O" form of a Cloaca.

5) We favour the suggestion of Edwards that the name Aerobacter should be chosen for the motile group; this would need the redefinition of the genus since the type species A. aerogenes disappears from it.

\section{REFERENCES}

1. Kauffmann, F. et al. Report on the Klebsiella (KlebsiellaAerogenes) Group. Internatl. Bull. Bact. Nomen. and Taxon. 4:75. 1954 .

2. Brooke, Marcus S. The differentlation of Aerobacter aerogenes and Aerobacter cloacae. Jour. Bact. 66:721. 1953.

3. Kauffmann, F. Enterobacterlaceae, 2nd Ed., E. Munksgaard, Copenhagen. 1954.

4. Moeller, V. Distribution of amino acid decarboxylases in Enterobacteriaceae. Acta Path. et Microbiol. Scand. 35:259. 1954 .

5. Moeller, $V$. Simplified tests for some amino acid decarboxylases and for the arginine dihydrolase system. Acta Path. ot Microblol. Scand. 36:158. 1955. 
Page 20

I N T E R N A I I O N A L B U L L E T I N

6. Edwards, P. R. and M. A. Fife. Studies on the KlebsiellaAerobacter group of bacteria. Jour. Bact. 70:382. 1955.

7. Levine, M. A statistical classification of the ColonCloacae group. Jour. Bact. 3:253. 1918.

Editorial Nate.

The serotype strains referred to in this paper include those described by Kauffmann (based on NCTC 5046-5056, Goslings and Snijders types A-F), Brooke, Edwards and Fife, and Edmunds. 